ENSAYOS

\title{
LA FORMACION DOCENTE BAJO UNA CONCEPTUALIZACION COMPREHENSIVA Y UN ENFOQUE POR COMPETENCIAS
}

The educational formation under a conceptualization comprehensive and an approach by competitions

\section{Arturo Barraza}

Barraza-upd@terra.com.mx

\begin{abstract}
Resumen
El presente trabajo se compone de tres partes: en una primera se aborda la formación profesional por competencias para hacer ver su principal limitación, la cual justifica y da origen al trabajo; en una segunda se realiza una discusión conceptual sobre el término formación para llegar a un concepto que guíe la reelaboración que se pretende hacer, y en una tercera se presenta una propuesta para conceptualizar la formación profesional de los docentes bajo un enfoque por competencias.
\end{abstract}

Palabras clave: formación, docente y competencia.

\begin{abstract}
The present work is made up of three parts: in one first part the professional formation by competitions is approached to make see its main limitation, which justifies and gives origin to the work, in one second part a conceptual discussion is made on the term formation to arrive at a concept that guides the reelaboration that is tried to do, and at a third moment a proposal appears to conceptualizar the professional formation of the educational ones under an approach by competitions.
\end{abstract}

Key words: formation, educational and competition. 


\section{PRESENTACION}

"En el mundo impera una tendencia en la formación profesional: la formación por competencias" (Cejas 2004:1); sin embargo, la traslación al campo de la formación de las nuevas tendencias y conceptos asociados a este enfoque no ha resultado tan inmediata como se quisiera y su implementación ha discurrido entre la crítica contumaz y el triunfalismo ingenuo.

"El debate sobre el significado, alcance y limitaciones de este nuevo enfoque ha sido y sigue siendo vivo e intenso" (Centro de Investigación y Documentación sobre problemas de la Economía, el Empleo y las Cualificaciones Profesionales $<<$ CIDEC $>>2004$ : 24), algunos autores (v. gr. Morfín 2000 y Tejada 2005) consideran que el concepto de competencia ha permitido superar modelos formativos precedentes, mientras que otros consideran que el enfoque por competencias es positivista, reduccionista, conservador y carece de base teórica (Prestón y Walker, citados por Gonczi y Athanasau 2000).

En el presente trabajo se asume una postura formativa, en sentido hegeliano, que parte de los siguientes supuestos:

- La construcción de un texto es un acto formativo.

- Como acto formativo, la construcción de un texto atraviesa por tres momentos: en un primer momento (el particular) el sujeto se reconoce ante el tema sobre el que va a escribir, la subjetividad se presenta como parte del tema a través de una serie de supuestos e hipótesis; en un segundo momento (el universal) el sujeto se reconoce ante los otros, ante el mundo objetivado en un conjunto de escritos y establece una relación con los autores de los textos que aborda para apropiarse o incorporar sus ideas; y en un tercer momento el sujeto retorna a sí mismo (el particular enriquecido), vuelve a reconocer su subjetividad a partir del mundo objetivado que ha incorporado y que ahora hace suyo y reconstruye.

- La construcción de un texto se convierte en un acto formativo cuando asume lo dado críticamente, cuando lo objetivado (acervo teórico existente) es negado por método y reconstruido por intención, cuando se toma una distancia de lo preexistente para fluidificarlo; el hacer de la negación un método, el no entregarse a la quietud es lo que hace que un proceso formativo sea impulsor del movimiento, sea capaz de "hacer fluido lo dado, mediante la oposición y la negación del presente" (Yurén 1993: 44).

- Hablar de negación es referirse a una gran variedad de relaciones: la diferencia, la oposición, la reflexión o la relación. "Puede indicar que una categoría es insuficiente o incoherente, y a veces las categorías mismas son contradictorias" (Spencer y Krauze 2002: 83).

- La construcción de un texto como acto formativo implica una actitud permanente de apertura dialéctica entre lo subjetivo y lo objetivo, entre el particular y el universal, entre la apropiación y la reconstrucción. La construcción de un texto no puede reducirse a ser un proceso transmisor que conserva lo dado, lo muerto.

Bajo estos postulados se inició la construcción del presente texto, el cual fue elaborado y reelaborado varias veces, como reflejo de la espiral dialéctica que acompañaba al proceso. 
Ya terminado, el texto se presenta con una estructura compuesta de tres partes: en una primera se aborda la formación profesional por competencias para hacer ver su principal limitación, la cual justifica y da origen al presente trabajo, en una segunda se realiza una discusión conceptual sobre el término formación para llegar a un concepto que guíe la reelaboración que se pretende hacer, y en una tercera se presenta una propuesta para conceptualizar la formación profesional de los docentes bajo un enfoque por competencias.

\section{LA FORMACION PROFESIONAL POR COMPETENCIAS}

El Concepto de Competencia Profesional surge en los años ochenta como una aportación central al debate que se vivía en los países industrializados sobre la necesidad de mejorar la relación de la educación con el sector productivo, relación caracterizada por la intención de impulsar una adecuada formación de la mano de obra.

$\mathrm{Su}$ desarrollo tuvo un impulso notable a partir de las experiencias generadas en países como Estados Unidos, Canadá, Australia y Reino Unido, al grado que países latinoamericanos como México, Colombia, Chile y Argentina, entre otros, se abrieron a este nuevo enfoque que constituye un punto de encuentro entre formación y empleo.

La literatura producida sobre este nuevo enfoque se ha centrado en la reseña de experiencias nacionales (Beltrán 2000; Hanson 2000, Kobinger 2000, y otros), en la construcción de marcos prescriptivos (Fletcher 2000, Saluda 2000, entre otros) y, en pocos casos, se ha intentado avanzar conceptualmente sobre la formación por competencias (Instituto Salvadoreño de Formación profesional <<INSAFORP>> 2001, CIDEC 2004 y Ruiz 2005).

La formación profesional vista desde el enfoque de competencias. La formación profesional por competencias se presenta bajo una dualidad, por una parte se habla de elaboración de programas de formación que tienen a la competencia como elemento central, mientras que por otra se habla de un proceso instruccional que sirve para mejorar la enseñanza y el aprendizaje y que tiene como elemento central la habilidad de aprender a aprender. En el primer caso se puede hablar del enfoque por competencias como un modelo curricular, mientras que en el segundo caso se le puede identificar como un modelo instruccional centrado en el aprendizaje.

Ante esta clasificación se hace necesario aclarar, aunque para algunos lectores resulte bastante obvio, que los dos modelos abordan tanto lo curricular como lo instruccional y su identificación en uno u otro modelo corresponde al énfasis que le otorgan a uno u otro aspecto; en el modelo curricular, a pesar de la centración en la elaboración de programas, se observan preocupaciones específicas sobre el aprendizaje (v. gr. Malpica 2000) y en el modelo instruccional centrado en el aprendizaje, más allá de los principios, ideas rectoras o estrategias que se esbozan, se hace referencia a lo curricular (v. gr. Ruiz 2005).

En el primero de los casos, el enfoque por competencias como modelo curricular, las características que definirían a un programa de formación elaborado en base a competencia serían los siguientes (INSAFORP 2001):

1. Los contenidos del curso se basan en tareas que se pueden medir.

2. Los objetivos de rendimiento de los participantes son especificados antes de la instrucción. 
3. El logro del participante se basa en demostrar la competencia.

4. La instrucción es individualizada.

5. El participante y el instructor son corresponsables del logro de las competencias.

6. El aprendizaje es guiado por la retroalimentación.

7. El tiempo de aprendizaje es flexible.

8. Se recomienda un programa de entrada/salida abierta.

9. La evaluación se realiza mediante una medición referida a criterio.

10. El instructor es un administrador y facilitador de la instrucción.

Por su parte, Tejada (2005) le asigna dos características centrales a los programas de estudio basados en un enfoque por competencias: flexibilidad y polivalencia:

Para que un plan formativo sea flexible y polivalente es preciso, hoy por hoy, pensar en diseños modulares, especificados en créditos y unidades didácticas. Ahora bien, estos módulos tienen que llenarse de contenido, en busca del logro de las competencias profesionales. No cabe duda que para que esto sea factible, los módulos serán unidades mínimas, pero con sentido, estructuradas en función del perfil profesional que se desee lograr (parr. 85).

Esta nueva forma de concebir la formación profesional "afecta igualmente a la configuración de la oferta educativo-formativa en sí misma, a su estructuración y planteamiento operativo" (CIDEC 2004: 27).

En el segundo caso, el enfoque por competencias como modelo instruccional basado en el aprendizaje, los aspectos estratégicos que definirían la esencia de una actuación pedagógica orientada al desarrollo o formación de competencias serían los siguientes (Ruiz 2005):

1. Componentes instruccionales (ejecución competente, el conocimiento del estado inicial del alumno, el aprendizaje y los procesos de transformación de un estado a otro y la evaluación y la orientación).

2. Aspectos inherentes a la génesis de formación de una competencia (amplia actividad mental, atender a lo óptimo, lograr la motivación y la ejercitación gradual y progresiva en busca de niveles de automatización).

3. Las ideas rectoras de un planteamiento constructivista (el alumno es el responsable último de su propio proceso de aprendizaje, la actividad mental constructiva del alumno se aplica a contenidos que poseen ya un grado considerable de elaboración y la función del docente es engarzar los procesos de construcción del alumno con el saber colectivo, culturalmente organizado).

4. Los principios metodológicos para la formación de competencias (significado por sobre estructuras, habilidades más allá del aula, realización de tareas en una visión integral y contextual, aula centrada en el alumno y el desarrollo de trabajo cooperativo).

5. Los objetivos intelectivos a lograr (favorecer el acceso a la información y su comprensión, favorecer el enriquecimiento asociativo, favorecer la integración y globalización de los contenidos, favorecer la aplicación a nivel de elaboración transformativa y aplicar un nivel elaborativo constructivo).

6. Las estrategias mediadoras de la planificación e intervención (externas e internas). 
Bajo estos dos modelos, la formación profesional por competencias puede ser definida, por una parte, como el desarrollo de programas ocupacionales, en los cuales se requieren conductas y estándares de rendimiento que son especificados antes de iniciar la instrucción, mientras que por otra, puede ser definida como un proceso instruccional, donde la intervención didáctica está centrada en el aprendizaje, basada en el rendimiento y referida a un criterio.

Posición crítica ante la formación por competencias. Más allá de las limitaciones o críticas que se le pueden hacer al enfoque de competencias, sea en su concepción general o en aspectos particulares del mismo, por el momento la atención del presente trabajo se centra en su carácter pragmático, que para algunos puede ser una ventaja ante los enfoques academicistas, pero que en este contexto se considera su principal limitación. El carácter pragmático del enfoque de competencias, que enfatiza el carácter práctico de la formación, suele presentarse con dos características que se encuentran asociadas al mismo; el utilitarismo y la inmediatez.

Este carácter pragmático le viene dado al enfoque de competencias por dos razones: su origen mismo y la concepción de formación presente en la empresa. Con relación al origen mismo se puede afirmar que:

Es a partir del auge de los modelos de desarrollo de la globalización cultural y la apertura económica que el término (competencia) toma fuerza en las políticas educativas, máxime para definir lo que se espera de la formación de los individuos para asumir los nuevos tipos de organización del trabajo y la inserción laboral (Ruiz 2005: 3)

La subordinación de este enfoque a fines eminentemente empresariales no ha podido ser superada a pesar de la pretensión, en el ámbito discursivo, de algunos autores al plantear fines más amplios como la ciudadanización, el desarrollo personal, etc.

Por otra parte, la manera en que es concebida la formación en el ámbito empresarial tiene ese carácter pragmático, ya que "la búsqueda de eficacia se revela como algo prioritario y se habla de ingeniería de la formación como conjunto coordinado de acciones para concebir, realizar y evaluar proyectos optimizando los costes de concepción y de gestión" (Moreno 2005: 2-3)

Este énfasis en la práctica, en el utilitarismo y en la inmediatez ha permeado todo el discurso del enfoque de competencias como se manifiesta en las siguientes citas:

"Lo importante es que el valor que se atribuye a los conocimientos no es poseerlos, sino hacer uso de ello" (CIDEC 2004: 26). La formación de competencias, desde una óptica diferente, se convierte en una alternativa necesaria ante las demandas del mundo laboral actual, porque el contenido de los empleos se modifica, se requiere de una polivalencia, polifuncionalidad y flexibilidad de los trabajadores, para que puedan cubrir varias dimensiones de la productividad (Ruiz 2005: 28).

"El propósito básico de la formación profesional por competencias es ayudar al participante a lograr las conductas requeridas o las competencias necesarias para tener éxito en el trabajo" (INSAFORP 2001: 11). Una noción de competencia en la que se privilegia el desempeño, entendido como la expresión concreta de los recursos que pone en juego el individuo cuando lleva a cabo una actividad, y que pone énfasis en el uso o manejo que el sujeto debe hacer de lo que sabe, no del conocimiento aislado, en condiciones en las que el desempeño sea relevante (Malpica 2000: 133). 
"El objetivo de la capacitación basada en competencias es desarrollar una fuerza laboral competente, con énfasis en la adquisición de habilidades prácticas necesarias en los lugares de trabajo, las cuales son definidas por los empleadores" (Saluda 2000: 146). La competencia profesional es el resultado de la integración, esencial y generalizada, de un complejo conjunto de conocimientos, habilidades y valores profesionales, que se manifiesta a través de un desempeño profesional eficiente en la solución de los problemas de su profesión, pudiendo incluso resolver aquellos no predeterminados (Forgas 2005; parr. 26).

Estas citas son solamente un ejemplo de cómo el discurso del enfoque de formación de competencias se mueve esencialmente, aunque no exclusivamente, en lo práctico, lo utilitario y lo inmediato, esto es, posee un carácter pragmático.

Una vez sustentada esta afirmación sobre el carácter pragmático del enfoque de competencias, es necesario retomar la línea discursiva enunciada en el primer párrafo de este rubro ¿por qué se considera una limitación?, la respuesta a esta pregunta es por que favorece una racionalidad de tipo instrumental.

Habermas (1993) distingue dos tipos de acciones susceptibles de racionalización: la acción orientada al éxito y la acción orientada al entendimiento.

La acción orientada al éxito o con arreglo a fines es en la que el actor se orienta exclusivamente por la consecución o logro de su objetivo global y somete a cálculo todas las demás consecuencias de la acción, considerándolas, simplemente, como condiciones secundarias del éxito al que aspira. "El éxito viene definido por la ocurrencia de un estado en el mundo, que en una situación dada puede ser causalmente producido mediante acción u omisión intencionadas" (Haberlas 1993: 384). Este tipo de acción se divide a su vez en dos: la acción instrumental y la acción estratégica.

Una acción orientada al éxito se denomina instrumental cuando se considera bajo el aspecto de la observancia, o cumplimiento, de reglas técnicas de acción que descansan en un saber empírico y cuya eficacia de intervención se evalúa en un estado físico u objetivo. La racionalidad que subyace a este tipo de acción se le denomina racionalidad instrumental.

Estas características de la racionalidad instrumental explican por qué la mayor parte del discurso que existe sobre el enfoque de competencias es de carácter prescriptivo y enfatiza los procedimientos y técnicas, sin olvidar su insistencia en el desempeño y la evidencia del desempeño.

Las implicaciones derivadas de este tipo de racionalidad son: a) se presupone la existencia de un solo mundo, el mundo objetivo, b) el nivel de racionalidad "se mide por la eficacia en la planificación del empleo de medios para fines dados" (Haberlas 2001: 233) y, c) su finalidad central es la solución de tareas técnicas y la construcción de medios eficaces.

Estas implicaciones conducen a la tecnificación de la realidad social y educativa, y sobre todo, en este segundo ámbito, orienta a una educación necrofílica que asume lo dado, que venera la norma fija y que anula el cambio y la reflexividad sustantiva basada en los fines.

Primer interludio: excurso para una generalización. Antes de continuar con el siguiente apartado es necesario hacer una aclaración sobre la intención de generalizar una crítica cuando, como lo deben estar pensando algunos lectores, el enfoque de competencias no se presenta como un bloque monolítico con una sola dirección. 
Ciertamente, la ambigüedad y la imprecisión acompañan al concepto de competencia, sin embargo, el concepto "sigue poseyendo un atractivo singular; parafraseando a Le Boterf (1996): la dificultad de definirlo crece con la necesidad de utilizarlo" (Tejada 2005; parr. 44). No obstante, de la multiplicidad de conceptos que se le han otorgado al término competencia se puede inferir que existen tres puntos de vista (Cejas y Pérez 2003):

- Punto de vista empresarial: la competencia vista en el desempeño eficiente del trabajador.

- Punto de vista psicológico: la competencia como una conformación psicológica compleja, que implica componentes motivacionales y afectivos del sujeto,

- Punto de vista del diseño curricular; la competencia como elemento articulador de la estructuración curricular y didáctica del sistema de conocimientos, habilidades, valores, aptitudes, actitudes y motivos que debe poseer un individuo para ejecutar sus tareas y su labor con el mínimo de requisitos exigidos en el contexto laboral, desde la visión de la escuela.

De estos tres puntos de vista el predominante es el empresarial como se demuestra en las definiciones proporcionadas por: Gallard y Jacinto (1995); Donna (2001); Ducci (en Guzmán e Irigoin 2000); Confederación Europea de Sindicatos (2002); Mertens (1996); OIT (en Briasco 2001); Ibarra (2000); Carrillo e Iranzo (en Sepúlveda 2002); Vargas, Casanova y Montanaro (2001); Programa Formujer (2003); Cuesta (2001); Cocca (2003); Wordruffe (1993); Sladogna (2000); Bunk (en Vargas, Casanova, Montanaro 2001); Ferrer (2002); Bolívar (2002); Reis (en Mertens 1996): Arbizu (2002); Barrios (2000); Spencer y Spencer (1993) y Páez (2002) (citados por Cejas 2004).

La predominancia de este punto de vista ha impactado al punto de vista curricular, mientras que el punto de vista psicológico se ha convertido en una herramienta más para sus fines, asimismo, nuevas líneas de discusión, v. gr: la Didáctica de la Educación Superior desarrollada sobre la base del Modelo Holístico Configuracional (Forgas 2003) y el trabajo por competencias en el prácticum (Tejada 2005) no pueden substraerse a la influencia del punto de vista empresarial. En ese sentido, la crítica realizada en el rubro anterior es totalmente extensible a la mayor parte de la literatura que existe sobre el tema de competencias o de formación por competencias.

\section{DISCUSION CONCEPTUAL SOBRE EL TERMINO FORMACION}

La formación ha sido, y es, objeto de diversas conceptualizaciones y referencias teóricas; esta situación se refleja en la multiplicidad de significados que presenta, por lo que la formación puede ser entendida como conjunto de actividades; como derecho de todo sujeto; dinámica de desarrollo personal; ponerse en forma; adquisición de conocimientos profesionales; proceso unificador de la evolución humana; proceso activo que requiere la mediación de otros; proceso de transformación; proceso social de desarrollo personal; un proceso eminentemente personal, etc. (Souto 1999).

Este panorama, nada halagüeño por cierto, conduce a concluir que el concepto de formación no es unívoco, por lo que se hace necesario una discusión conceptual que 
organice y le otorgue coherencia al campo, y para tal efecto se considera ineludible una revisión de la literatura existente en el campo.

Una primera revisión de la literatura permite reconocer que el término formación es abordado en cinco campos de estudio: la filosofía, la psicología laboral, el psicoanálisis, la pedagogía y la formatividad.

La formación en el campo de la filosofía. El término formación es uno de los conceptos básicos, y esencialmente constituyente del humanismo; proviene de la Edad Media, pasó por el Barroco y se integró al Humanismo cuando Herder (citado por Gadamer 1996) preconizó el ideal de la "formación del hombre"; el Humanismo es un modo de ver la realidad, una perspectiva que considera a la dignidad humana como criterio último de las valoraciones y normas y como el horizonte al que deben orientarse las acciones, si es que se desea una vida que valga la pena ser vivida.

La formulación más acabada, o en término de Gadamer (1996) más profundamente desarrollada, del concepto de formación se puede encontrar en los trabajos de Hegel (citado por Yurén 2000), quien conceptualiza a la formación como bildung, lo que implica que la persona: a) se apropie de las normas y los órdenes institucionales existentes y los cumpla, b) se apropie de la cultura de su tiempo, y c) se configure a sí misma como sujeto en virtud de que crea, recrea o renueva la cultura y los órdenes sociales. Esta última implicación es la que considera Hegel (citado por Ginzo 1998) como formación, o más estrictamente como el "concepto dialéctico de formación espiritual" (Abbagnano y Visalberghi 1993).

Este concepto de formación presenta tres características centrales: la idea de incompletud del sujeto en formación, el papel activo del sujeto en formación en un marco de intersubjetividad y la formación como retorno sobre sí mismo.

El plantear la formación en términos hegelianos parte del hecho de reconocer la idea de incompletud en el hombre por su condición evolutiva; Tanto la "especie" como el "individuo" humanos comienzan existiendo como espíritu u hombre naturales. Pero si bien el animal es por naturaleza tal como debe ser, el hombre, por el contrario, no lo es. Sólo la formación y la educación hacen al hombre tal como debe ser. Por eso se refiere Hegel a la educación como un segundo nacimiento. Frente al carácter estático de la naturaleza, el espíritu necesita de la educación para alcanzar su auténtica realización (Ginzo 1998: 33-34).

Bajo esta concepción, Hegel (citado por Ginzo 1998) se separa del romanticismo naturalista de corte rousseaniano y se muestra sensible a lo que le falta a la infancia, en cuanto etapa, hacia la edad adulta. En ese sentido, el bildung (configuración o construcción) parte del supuesto de que el hombre al nacer, se inserta en un mundo dado, aunque no acabado, por lo que un tramo de su formación consiste en apropiarse de ese mundo circundante y situarse en él, empero, esta apropiación del mundo, sea en términos de socialización (inciso a) o enculturación (inciso b) tiene como condición sine qua non que todo lo que el individuo aprende o interioriza, lo hace en relación con los otros. Esta característica es identificada por Yuren (2000) como el momento de la intersubjetividad, pero a su vez, es ampliada por la autora al integrar la acción comunicativa, en términos habermasianos, como aspecto central a cubrir en la interacción.

Lograr reconocerse en el otro y ser capaz de distanciarse de sí mismo en un acto de apertura hacia los demás para entender sus ideas y sus pensamientos y poder retornar 
a sí mismo, a sus ideas y conocimientos, es el propósito final de la interacción; esta característica, en otros términos, consistiría en que del particular (espíritu subjetivo), se transita a lo universal (espíritu objetivo), para regresar a un particular enriquecido por lo universal (espíritu absoluto), el cual puede crear y recrear lo universal en un acto de apertura eminentemente dialéctico (inciso c).

Este retorno sobre sí mismo es lo que permite el tránsito de una "conciencia de sî" a una "conciencia para sî" o autoconciencia.

La formación en el campo de la psicología laboral. La formación del personal es un rubro recurrente en el campo de la psicología laboral o del trabajo, por un lado suele presentarse su concepción y características, y por el otro, se le ve como un proceso compuesto de varias etapas.

En lo concerniente a su concepción se pueden encontrar definiciones que enfatizan a) su carácter procesual, v. gr. "desde un enfoque estratégico, la formación es un proceso integrado en la gestión de recursos humanos para servir a la estrategia de la empresa. Por eso parte de un diagnóstico de necesidades empresariales a las que se dan respuesta con las acciones formativas que deben valorarse en función de su impacto en el desempeño de su puesto de trabajo" (Guillén y Guil 1999: 230), b) sus componentes estructurales, v. gr. "la formación del personal se define como el proceso sistemático de adquisición de actitudes, conceptos, conocimientos, funciones o destrezas que dan lugar a una mejora continuada de los niveles de rendimiento laboral" (García, Rubio y Lillo 2003: 126), o c) las actividades que involucra, v. gr. "la formación profesional es una actividad cuyo objeto es descubrir y desarrollar las aptitudes humanas para una vida activa, productiva y satisfactoria. En función de ello, quienes participan de actividades de formación profesional deberían poder comprender individual o colectivamente cuánto concierne a las condiciones de trabajo y al medio social, e influir sobre ellos".

Continuando con la misma línea de razonamiento, es posible afirmar que la formación profesional son simultáneamente, tres cosas:

- $\quad$ Es una actividad de tipo educativo (...),

- Es una actividad vinculada a los procesos de transferencia, innovación y desarrollo de tecnología (...),

- La formación profesional es un hecho laboral y, como tal, posee un lugar indiscutible dentro de las relaciones de trabajo (Casanova 2003: 10).

Con relación a las etapas que involucra la formación se pueden observar similitudes, más allá de la terminología utilizada, como queda de manifiesto en el cuadro 1 (ver página siguiente).

Más allá de la coincidencia o no que se tenga en la identificación de las diferentes etapas que involucra la formación, el énfasis en su carácter procesual constituye un aporte importante al campo de la formación.

Las definiciones, presentadas en párrafos anteriores, y las etapas esquematizadas en el cuadro 1, permiten afirmar que lo común en las diferentes formas de abordar la formación, en este campo, es su preocupación, comparativamente mayor que otras formas de educación, por los contenidos y los métodos de dicha formación, en lo general o 


\section{Cuadro 1}

Etapas de formación en dos perspectivas

\begin{tabular}{|l|l|}
\hline Guillén y Guil (1999) & García, Rubio y Lillo (2003) \\
\hline $\begin{array}{l}\text { Detectar las necesidades (reales y previstas) } \\
\text { de la organización. }\end{array}$ & Análisis de las necesidades de formación \\
$\begin{array}{l}\text { Determinar las posibilidades de respuesta que } \\
\text { la formación puede ofrecer. } \\
\text { Disponer los medios posibles. }\end{array}$ & Definición de los objetivos de la formación \\
Diseñar las acciones pertinentes. & $\begin{array}{l}\text { Desarrollo de medidas criterio para evaluar la } \\
\text { formación } \\
\text { Garantizar la calidad de la impartición. }\end{array}$ \\
$\begin{array}{l}\text { Planificación del contenido de la formación } \\
\text { Diseño de los métodos y de los materiales } \\
\text { Eue hay que utilizar en la formación }\end{array}$ \\
mativa) y su impacto en la realidad laboral. & $\begin{array}{l}\text { Adaptar el programa de formación a los que } \\
\text { van a ser formados } \\
\text { Evaluación de la eficacia del programa de } \\
\text { formación }\end{array}$ \\
\hline
\end{tabular}

Fuente: Elaboración propia.

en algunas de sus etapas, por un lado, y los cambios que se operan en el mundo de la producción y el trabajo, por otro. Esta característica le da su carácter instrumental y pragmático vinculado a objetivos o fines inmediatistas.

Segundo interludio: una aclaración necesaria. Antes de continuar con el siguiente rubro que aborda la formación en el campo del psicoanálisis, es necesario realizar una aclaración al respecto.

Davini (1997) reconoce tres líneas de pensamiento en el campo de la formación de docentes: la producción francesa, los aportes de la bibliografía de habla inglesa y la línea latinoamericana. De estas tres líneas de pensamiento cobra relevancia, por los objetivos del presente trabajo, la producción francesa, la cual no se presenta, como algunos suelen pensar, como un bloque monolítico, sino que incluye en su seno diferentes perspectivas.

De las diferentes perspectivas que coexisten al interior de esta línea de pensamiento, se abordarán dos que aportan elementos a la discusión que se desarrolla en el presente trabajo: el Psicoanálisis de la Formación (Filloux 1996) y la Pedagogía de la Formación (Ferry 1997).

La formación en el campo del psicoanálisis. La influencia de la teoría psicoanalítica en el campo educativo es innegable y su presencia se extiende desde los primeros trabajos con educadores de Anna Freud (citada por Gilbert 1977) en la década de los treinta, hasta la tentativa de crear una pedagogía psicoanalítica (Schraml 1970).

Más allá de esta trayectoria histórica, es posible identificar dos niveles en que se da la relación del psicoanálisis con la formación: a) en un primer nivel se aplica el saber psicoanalítico a la formación, por lo que el psicoanálisis se convierte en un discurso 
prescriptivo, b) en un segundo nivel se utiliza el saber psicoanalítico para explicar o interpretar estudios o investigaciones realizadas en el campo de la formación, por lo que el psicoanálisis se constituye en un discurso explicativo. En este segundo nivel se sitúa el psicoanálisis de la formación y centra su atención en los aspectos relacionales del aula y a partir de ellos ha intentado formular un modelo teórico, el cual es solidario a un concepto de formación.

Filloux (1996) asume y hace suya la concepción de formación como retorno sobre sí mismo de Hegel (vid supra rubro la formación en el campo de la filosofía), pero en su perspectiva, este retorno contiene pensamientos, sentimientos y percepciones sobre uno mismo, por lo que formarse implica cuestionarse, buscar en lo profundo, en los deseos y en las fantasías para encontrar aspectos nuevos y/o zonas de ignorancia, de rechazo o de negación, que el reflejo en el otro provoca y muestra.

Bajo esta concepción, la formación se concreta en la movilización de los procesos psíquicos primarios (emociones, fantasías, deseos, defensa, etc.); en término de Souto (1999), "los cambios integrales del sujeto requieren de una movilización de las emociones y de un trabajo sobre ellas" (p. 47), es por eso que la formación debe ser definida como un proceso eminentemente personal, pero que tiene como condición de realización, las relaciones entre las personas.

Estas relaciones entre el formante y el sujeto en formación ponen en evidencia lo que Freud (citado por Filloux, 1996) llamaba la otra escena. En este otro escenario el punto de interés son los sujetos en relación, el formante y el sujeto en formación, y la relación entre ambos se constituye en un "dialogo inconsciente entre los inconscientes" (Filloux 1996: 28), este diálogo es mediado, entre otros aspectos, por fantasmas y deseos.

En la relación de formación se presentan todo tipo de fantasmas (fabricación de representaciones con elementos del inconsciente) que salen a la luz y le dan un carácter totalmente particular a la relación. Hay formantes que querrán ser buenas madres, otros malas madres, otros formantes tendrán una fantasía de paternidad y otros tantos tendrán la fantasía del modelaje, el denominado fantasma pigmalión.

Los fantasmas son el motor de la acción del formante, le ayudan a actuar; sin embargo, éstos pueden ser o no adecuados, por lo que se les requiere administrar, controlar y regular y esto se logra en el retorno sobre sí mismo, el cual permite conocer los fantasmas y por ende, trabajar sobre ellos.

Otro aspecto importante en la relación formante y sujeto en formación es el deseo. Algunos formadores piensan que su labor se circunscribe a la instrucción, sin embargo, en lo más profundo ¿cuál es su deseo?, ¿qué es lo que realmente quiere hacer? ¿es formar? ¿instruir? ¿tener poder? ¿ser importante? ¿ser amado? O a veces, por qué no, ¿ser odiado?

El formante regularmente suele enfrentarse a tres deseos: a) el deseo de saber que es a la vez un deseo de no saber, ya que saber demasiado crea culpa, b) el deseo de un saber sobre el deseo, y c) el deseo de amar y/o de ser amado. Este último deseo se encuentra presente tanto en el formante como en el sujeto en formación.

Este cruce de deseo y fantasmas, en particular, o los efectos del inconsciente, en lo general, se ven reflejados en las relaciones de transferencia y contra transferencia, en la pulsión de apoderamiento, o en la ambivalencia, identificación y erotización de las relaciones mismas; en ese sentido, la transformación del sujeto surge tanto de la temática como de los procesos grupales y de las relaciones transferenciales y de identificación 
que se van produciendo en el espacio que se comparte; por lo tanto, los formantes deben estar atentos a la interacción permanente entre lo que tiene que ver con el orden del deseo, de lo afectivo, del odio, del miedo, de la culpa, de la angustia y también lo que surge del inconsciente y lo que tiene que ver con lo intelectual.

La formación en el campo de la pedagogía. El término formación aparece recurrentemente en obras de carácter pedagógico, sin embargo, su sola mención no implica que se tenga claridad sobre su significado; esta situación puede observarse en múltiples obras donde el concepto formación es reducido a ciertos contextos y/o prácticas institucionales o a ciertos dispositivos de formación (v. gr. Davini 1997, Imbernón 1997, Alanis 2000 y Aguerrondo y Pogré 2001).

Una de las excepciones a esta regla es el trabajo desarrollado por Gilles Ferry, quien, principalmente, a través de dos de sus obras ha constituido a la formación en su objeto de estudio.

Las obras El trayecto de la formación. Los enseñantes entre la teoría y la práctica y Pedagogía de la formación muestran las preocupaciones de Ferry por dilucidar el concepto de formación y la relación teoría-práctica que suele acompañarle. Por el objetivo que se persigue en el presente trabajo la atención se centra en la segunda y específicamente en el concepto de formación que propone el autor.

Formar es algo que tiene que ver con la forma, por lo tanto formarse tiene que ver con adquirir una forma, la cual, a su vez, posibilita actuar y reflexionar para perfeccionar esa forma.

Esa forma, si está enfocada al campo profesional, consistiría en una forma que tendría, como intencionalidad central, cumplir con las tareas requeridas para ejercer esa profesión. Adquirir esa forma implica la adquisición de conocimientos, habilidades, ciertas representaciones del trabajo a desarrollar o de la profesión que se va a ejercer, la concepción del propio rol profesional, etc.

¿Cómo es que se adquiere esa forma? Ferry (1997) responde a esta pregunta afirmando que es el individuo el que se forma, o transita de forma a forma; sin embargo, se hace necesario reconocer que el sujeto se forma a sí mismo, pero se forma sólo por mediación.

Las mediaciones son variadas y diversas. Los formadores son mediadores humanos, lo son también las lecturas, las circunstancias, los accidentes de la vida, la relación con los otros... Todas estas son mediaciones que posibilitan la formación, que orientan el desarrollo, la dinámica del desarrollo en un sentido positivo (Ferry 1997: 55).

La formación, así entendida, conduciría a establecer que el papel del formador es ayudar, a través de mediaciones, a que el sujeto en formación se desarrolle trabajando sobre sí mismo. Para el logro de tal cometido se requieren situaciones que cubran tres condiciones: tiempo, lugar y relación con la realidad.

Trabajar sobre sí mismo sólo se puede hacer en los lugares y tiempos establecidos para tal propósito y la relación con la realidad se logra al establecer una distancia con respecto a ella y representársela para trabajar sobre ella, ya que cualquier acción formativa tiene como materia prima la representación de esa realidad y no la realidad misma.

La formación en el campo de la normatividad. Ante el uso inadecuado del concepto formación o de las teorías, para conceptualizarlo, provenientes de campos de acción 
como la enseñanza, la terapia o el management, Honoré (1980) propone constituir a la formación como objeto de estudio y reagrupar los trabajos que existen al respecto en un nuevo campo de estudio denominado formática o formatividad.

Este nuevo campo de estudio parte de las siguientes premisas: a) la formación es una función evolutiva, b) la formación pone en juego la dimensión teleológica de los fenómenos humanos, c) el conocimiento del proceso de formación necesita una teorización en tres planos: el espacio relacional, el tiempo cambio y la energía organizadora, y d) la formación se manifiesta en un conjunto de fenómenos que constituyen el campo de la formatividad.

Bajo estas premisas, y en el marco de la formatividad como campo de estudio, se puede considerar a la formación como una experiencia, o función evolutiva, que implica "un proceso de diferenciación y de activación energética que se ejerce a todos los niveles de la vida y del pensamiento" (Honoré 1980: 105).

La diferenciación se define como el proceso mediante el cual dos cosas parecidas llegan a ser diferentes, proceso que se da al establecer en ellas una relación de alteridad más allá de sus elementos idénticos. Esta diferenciación se da entre interioridad y exterioridad, entre formante y sujeto en formación, entre sujeto y objeto, entre pasado y porvenir, etc.

La diferenciación se presenta bajo un doble aspecto: la creación de la diferencia y de la identidad.

La activación energética puede ser definida como "el proceso por el cual la energía se transforma irreversiblemente, y de manera discontinua, desde las modalidades reactivas elementales de la vida hasta las manifestaciones espirituales más elaboradas de la experiencia humana" (Honoré 1980: 116); ese proceso corresponde a la elaboración de una información de complejidad significativa creciente, por lo que se puede hablar de niveles de información o de reflexión que obedecen también a un proceso de diferenciación que conduce a la dicotomía inorganizado-organizado.

Las premisas y el concepto, esbozados anteriormente, necesitan un correlato práctico para asegurar a la formación un desarrollo, en ese sentido es que se plantean ciertas condiciones para la formación:

1. Superación de la separación entre formación personal y la formación profesional.

2. Alternancia del tiempo de formación profesional y de tiempo de formación interprofesional.

3. Alternancia de tiempo de formación en la institución y de tiempo de formación fuera de la institución.

4. La formación debe ser por todas partes instituyente de formación y realmente continua.

5. Una política de formación debe ser una política de intervención.

Hacia una conceptualización comprehensiva. Una conceptualización, o en estricto sentido del término una reconceptualización, no puede partir de la nada, sino que se realiza sobre la información precedente, en ese sentido, utilizando la información relativa a cómo es abordada la formación en los diferentes campos de estudio se procede a identificar aquellos elementos que pueden dar origen a las ideas fuerza que articulen un concepto comprehensivo de formación. 
En primer lugar, es necesario descartar el concepto de formación que se tiene en el campo de la psicología laboral, ya que comparte el carácter pragmático que se le adjudica a la formación profesional por competencias, situación que se desea superar; sin embargo, el énfasis en el aspecto procesual de la formación es un elemento que puede ser rescatable.

En segundo lugar, la intersubjetividad y el retorno sobre sí mismo son dos elementos que se consideran indispensables retomar de la perspectiva filosófica para conceptualizar a la formación; cosa distinta ocurre con la noción de incompletud que se considera un elemento innecesario.

Es menester marcar, como tónica general del presente trabajo, el distanciamiento con los aspectos evolutivos del ser humano, ya que la creencia en una lógica evolutiva lineal es una visión solidaria del proyecto de la modernidad y este proyecto carece de sentido en una época donde la incredulidad, el deslizamiento de las fronteras e identidades y el carácter flotante del signo (vid Lyotard, Vattimo, Laclau, Derrida, Castoriadis, Kuhn y Lacán, citados por Barraza 2004) hablan de la erosión del carácter absoluto, atemporal y totalizador de los discursos y categorías.

En tercer lugar es necesario reconocer lo atractivo que puede llegar a ser el discurso psicoanalítico, no obstante se puede vivir con él, sin él y a pesar de él, por lo que su propuesta de contenido (procesos psíquicos primarios) para la intersubjetividad y el retorno sobre sí mismo puede ser prescindible o, en el mejor de los casos, ubicada en un segundo término.

Más allá del reduccionismo teórico que implicaría adoptar la perspectiva psicoanalítica, sus principales limitaciones se tendrían en el terreno práctico del proceso formativo: personal que formaría a los formantes, tiempo de formación, etc.

En cuarto lugar, con relación a la postura pedagógica, se puede afirmar que la propuesta de contenido a abordar en la formación es reduccionista porque se circunscribe al campo profesional y su concepto de mediación es tan amplio como impreciso. En este segundo punto baste recordar que el mismo Vygotski (citado por Leontiev 1995) superó la mediación de corte instrumental a favor de una mediación semiótica.

En quinto lugar, la propuesta de la formatividad como campo de estudio se torna interesante, aunque su discurso conceptual presenta un alto grado de opacidad, amen de algunas imprecisiones: sin embargo, para el propósito que se persigue en el presente trabajo se considera que la apuesta por la diferenciación es reduccionista y puede ser asimilada en la perspectiva hegeliana sin ningún problema, mientras que el concepto de activación energética es poco claro y por consecuencia posee poca capacidad heurística, por lo que es prescindible.

Como resultado del anterior análisis se pueden establecer las siguientes ideas fuerza para un concepto de formación más comprehensivo:

- La formación es un proceso dialéctico eminentemente personal.

- La trascendencia de la subjetividad se realiza al ponerse el sujeto en formación en relación con los otros y posteriormente al realizar el retorno sobre sí mismo.

- La relación con los otros implica esencialmente apropiación y el retorno sobre sí mismo implica necesariamente reconstrucción.

- El contenido sobre el que trabaja el sujeto en formación son los órdenes socioinstitucionales y la cultura, sea de una sociedad en su conjunto, o de una organización laboral en lo específico. 
- Las prácticas laborales y/o profesionales atienden al proceso de formación en sus dos vertientes: apropiación y reconstrucción.

- La ayuda al proceso de formación la realiza el formante a partir de cuatro etapas: identificación de necesidades de formación (fase diagnóstica), elaboración de los programas de formación (fase curricular), desarrollo de los programas de formación (fase de actuación del formante y del sujeto en formación) y evaluación de los programas de formación y de los aprendizajes desarrollados (fase de resultados).

\section{UNA PROPUESTA DE COMPETENCIAS GENERICAS PARA LA FORMACION DE LOS DOCENTES}

El contenido de una formación básica sería, sin lugar a dudas, los órdenes socioinstitucionales y la cultura, sin embargo, en la formación profesional su contenido serían las prácticas profesionales; en el caso de la formación docente, ésta se abocaría, esencialmente, al desarrollo de esa práctica profesional denominada docencia.

¿Cómo formar a un sujeto para el desarrollo profesional de la docencia? La respuesta a esta pregunta se encuentra en una formación, entendida de manera comprehensiva, de competencias profesionales.

Las competencias profesionales: a la búsqueda de un concepto. El origen del término competencia es adjudicado en dos contextos diferentes, a dos autores diferentes: por un lado, Cejas (2004) refiere que el concepto de competencia aparece en los años 70, especialmente a partir de los trabajos de McClelland en la Universidad de Harvard, mientras que por otro, Ruiz (2005) lo deriva de la lingüística, especialmente de los trabajos de Chomskii.

La postura en el presente trabajo es considerar que el origen que le atribuye Cejas (2004) es el adecuado, ya que su esencia está ligada a la noción de desempeño, la cual es central para entender lo que es una competencia, mientras que en el segundo origen atribuido se tiene un problema con la connotación del término competencia, ya que Chomski (citado por Ruiz 2005) identifica dos componentes lingüísticos: competencia y actuación o realización (performance), mientras que la competencia se refiere "a un conocimiento personal, no acabado, (...) La suma de todas las estructuras elementales y de las reglas de combinación que ha almacenado, y que puede movilizar si tiene necesidad de hacerlo, es una abstracción. La realización es el empleo que cada quien hace de ese sistema de funcionamiento" (Aimard 1987: 28-27).

En ese sentido, el término competencia que se maneja actualmente, cuando se habla de este enfoque, estaría más ligado al componente de la actuación.

Más allá del origen del término, lo que sí se puede afirmar, recuperando la idea expresada en el primer interludio, es que la ambigüedad y la imprecisión acompañan al concepto de competencia, situación que es trasladable a sus derivaciones como sería el caso de la competencia laboral o profesional que en muchas ocasiones son manejadas indistintamente (Cejas 2004).

Bajo ese contexto discursivo se puede encontrar que la competencia es definida como capacidad productiva, aptitud de un individuo, capacidad real u objetiva del individuo, capacidades adquiridas, habilidad, habilidad multifacética, operacionalización, operacio- 
nes mentales, cognitivas, socioafectivas y psicomotoras, conjunto de comportamientos socioafectivos y habilidades cognoscitivas, psicológicas, sensoriales y motoras, conjunto de conocimientos teóricos, habilidades, destrezas y actitudes, un sistema de atributos en estrecha vinculación, un sistema de conocimientos, conceptuales y de procedimientos organizados en esquemas operacionales, una compleja estructura de atributos, combinación integrada de conocimientos, habilidades y actitudes, un sistema de conocimientos, habilidades, actitudes, valores, motivos, aptitudes y capacidades, engloba no sólo las capacidades requeridas para el ejercicio de una actividad profesional, sino también un conjunto de comportamientos, facultad de análisis, toma de decisiones, transmisión de información, etc., es la construcción social de aprendizajes significativos y útiles, compleja integración de atributos, etc.

Después de este listado, no exhaustivo, pero sí ilustrativo la pregunta sería ¿qué es una competencia?

- ¿es una capacidad, una aptitud, una habilidad, una habilidad multifacética, o

- un conjunto de operaciones mentales, un conjunto de comportamientos, un conjunto de conocimientos, habilidades, destrezas y actitudes, un conjunto de comportamientos, facultad de análisis, toma de decisiones, transmisión de información, etc., o

- un sistema de atributos, un sistema de conocimientos, conceptuales y de procedimientos organizados en esquemas operacionales, un sistema de conocimientos, habilidades, actitudes, valores, motivos, aptitudes y capacidades, o

- una compleja estructura de atributos, o

- una construcción social de aprendizajes significativos y útiles, etc.?

Un primer filtro, para empezar a decantar este conjunto de definiciones, es tomar la decisión de atender a una de las características centrales de lo que es una competencia: otorgar un significado de unidad.

En las definiciones presentadas es posible identificar tres tipos de definiciones: aquellas que remiten a un significado unitario simple (capacidad, habilidad, etc.), las que remiten a un significado unitario complejo (estructura, sistema, etc.) y las que refieren a un significado plural (conjunto); en primera instancia, si se atiende a la idea de un significado de unidad, la apuesta debe de girar hacia las definiciones que remiten a un significado unitario, sea éste simple o complejo, ya que aquellas que remiten a un significado plural ofrecen una visión de yuxtaposición y provocan en la práctica grandes errores (v. gr. la Licenciatura en Intervención Educativa de la Universidad Pedagógica Nacional que establece competencias de saber, por un lado, competencias de saber hacer por otro lado, etc.).

Bajo ese filtro, la diversidad de respuestas a lo que es una competencia se reduce a términos como capacidad, aptitud, habilidad, atributo, estructura, sistema, etc.; la selección del término más adecuado, a partir de una discusión conceptual que establezca los límites y alcances de cada concepto, es una actividad que rebasa los límites del presente trabajo, por lo que no se intentará realizar esa discusión, pero ante la necesidad de contar con una conceptualización del término se considera más conveniente remitirse a las perspectivas conceptuales tradicionales.

Regularmente han sido tres las perspectivas conceptuales utilizadas para definir lo que es una competencia: como lista de tareas, como conjunto de atributos y como relación holística o integrada (Gonczi y Athanasou 2000). 
Desde la primera perspectiva la competencia se analiza con relación a las tareas independientes que se llevan a cabo. Esta perspectiva es identificada por Cejas y Pérez (2003), como el punto de vista empresarial y por Saluja (2000) como el enfoque del Reino Unido.

La segunda perspectiva identifica a la competencia como los atributos generales indispensables para el desempeño efectivo. Esta perspectiva es identificada por Cejas y Pérez (2003) como el punto de vista psicológico, y por Saluja (2000) como el enfoque de los Estados Unidos.

La tercera perspectiva conceptual combina los dos enfoques anteriores e integra atributos y tareas. Esta perspectiva es la que se acepta para el presente trabajo, pero antes de continuar es necesario hacer una aclaración: algunos lectores tal vez estén visualizando una contradicción en la línea argumentativa que se viene desarrollando, ya que en un primer momento se eligió la opción de definiciones orientadas a un significado unitario y por otra parte se abordan las perspectivas conceptuales que, aparentemente, orientan a significados plurales, sin embargo no existe tal contradicción, ya que la presentación en términos plurales de las perspectivas conceptuales es un problema de relación entre el significado y el contexto del discurso, ya que párrafos más adelante Gonczi y Athanasau (2000) ofrecen ejemplos de cómo concebir la competencia, la cual es definida en términos unitarios, sobre todo en el caso de la perspectiva conceptual holística: "la competencia se concibe como una compleja estructura de atributos necesarios para el desempeño en situaciones específicas" (p. 275).

Competencias genéricas para la formación de los docentes. Para definir las competencias necesarias para la formación de los docentes se toma como base el modelo de competencias profesionales integrales (Huerta, Pérez y Castellanos 2000), el cual establece tres niveles: las competencias básicas, las genéricas y las específicas, y su rango de generalidad va de lo amplio a lo particular.

Las competencias básicas son las capacidades intelectuales indispensables para el aprendizaje de una profesión; las cuales son adquiridas en los niveles educativos previos (por ejemplo el uso adecuado de los lenguajes oral, escrito y matemático).

Las competencias genéricas son la base común de la profesión y pueden o no referirse a situaciones concretas de la práctica profesional, siempre y cuando éstas requieran respuestas complejas.

Las competencias específicas son la base particular de la práctica profesional y están vinculadas a condiciones específicas de desempeño..

En el caso de los docentes se puede afirmar que: a) las competencias básicas ya han sido formadas en los niveles educativos previos, b) las competencias genéricas deben de responder en su esencia a un modelo de formación, y c) las competencias específicas se pueden derivar de estrategias de construcción de competencias como el Taller de Análisis Funcional o el Taller Dacum.

Con base en estos supuestos el presente trabajo se aboca a construir las competencias genéricas que respondan a una conceptualización comprehensiva de la formación, en ese sentido es posible identificar dentro de esta conceptualización tres procesos: uno objetivo (relación del sujeto con su entorno), uno intersubjetivo (relación del sujeto con otros sujetos) y uno intrasubjetivo (relación del sujeto consigo mismo). 
El proceso objetivo se ubica en el momento de la apropiación, el proceso intersubjetivo se ubica en el momento de la intersubjetividad y el proceso intrasubjetivo se ubica en el momento del retorno sobre sí mismo.

Las competencias y atributos referidos al momento de la apropiación que deberían de desarrollar los formantes son los siguientes:

\section{Cuadro 2}

Competencias y atributos referidos al momento de la apropiación

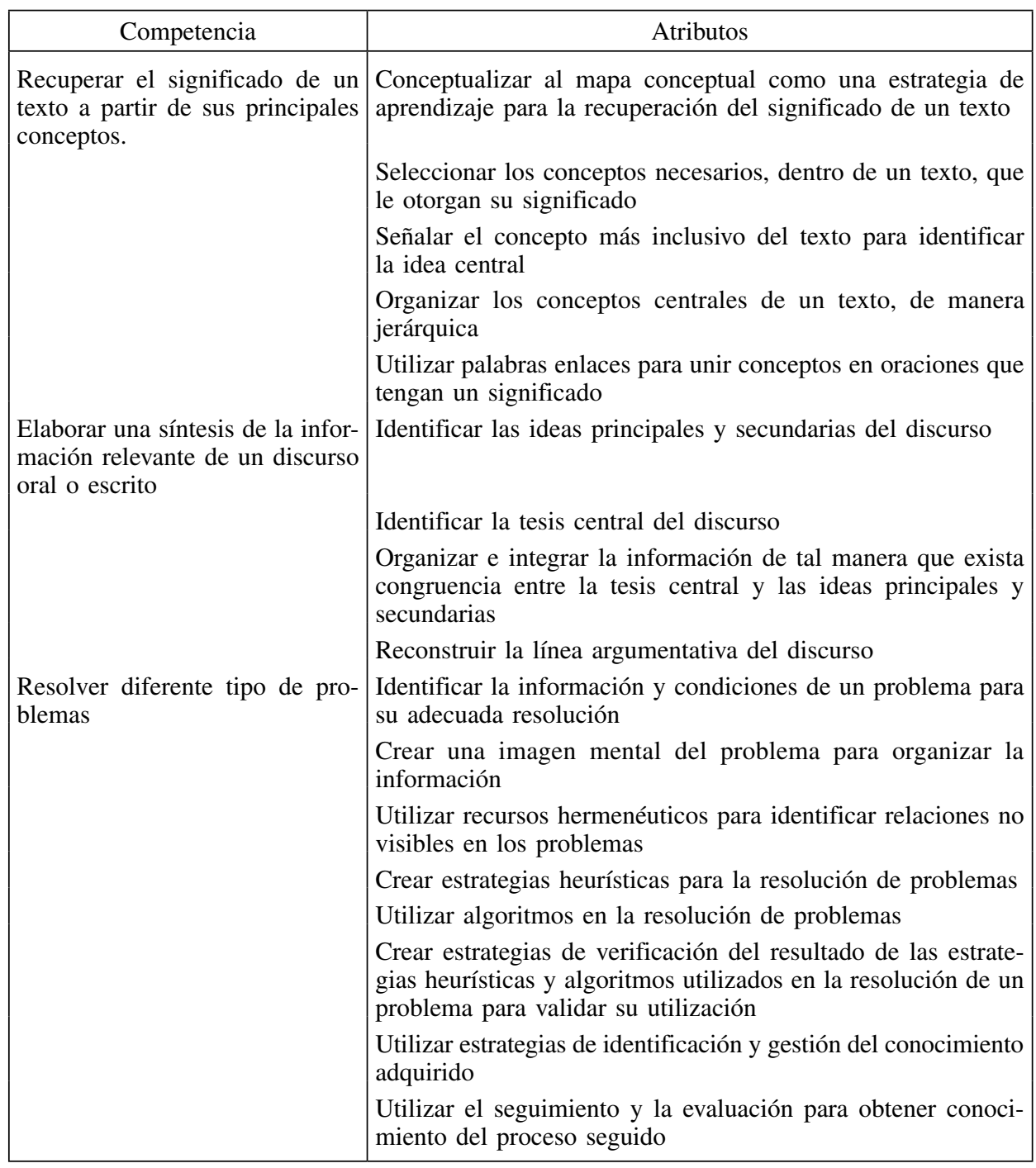

Fuente: Elaboración propia. 
Las dos primeras competencias conducirían a la apropiación de contenidos conceptuales, mientras que la tercera conduciría a la construcción y apropiación de contenidos procedimentales.

Con relación al momento de la intersubjetividad, las competencias y atributos que el formante debería de desarrollar son los siguientes:

\section{Cuadro 3}

Competencias y atributos referidos al momento de la intersubjetividad

\begin{tabular}{|l|l|}
\hline COMPETENCIA & ATRIBUTOS \\
\hline Participar en trabajos colaborativos & $\begin{array}{l}\text { Mostrar disposición para trabajar con otras personas } \\
\text { Estimular a sus compañeros a establecer orientaciones de } \\
\text { actuación } \\
\text { Establecer los elementos comunes en la comprensión de } \\
\text { problemas o situaciones dadas } \\
\text { Crear relaciones de apoyo y respeto con los compañeros } \\
\text { de trabajo } \\
\text { Comunicar con precisión y claridad sus pensamientos } \\
\text { Alentar la construcción de pautas de funcionamiento para } \\
\text { mantener las relaciones de trabajo } \\
\text { Utilizar el lenguaje no verbal y la proxémica para generar } \\
\text { confianza en sus relaciones con los demás } \\
\text { Valorar la estructura organizativa del trabajo en equipo como } \\
\text { la más idónea para un trabajo colaborativo } \\
\text { Favorecer una interdependencia positiva en la atribución de } \\
\text { dizaje }\end{array}$ \\
$\begin{array}{ll}\text { los resultados obtenidos } \\
\text { Utilizar el conocimiento de cada uno de los miembros de } \\
\text { una comunidad para construir un conocimiento colectivo } \\
\text { Articular, con los demás participantes de una comunidad, } \\
\text { los objetivos que persiguen y los términos en que van a } \\
\text { juzgar su nivel de éxito } \\
\text { Participar en el uso de procesos metacognitivos de carácter } \\
\text { colectivo } \\
\text { Alentar la superación de los límites que, a nivel de cono- } \\
\text { cimiento y aptitudes, tiene una comunidad } \\
\text { Negociar el significado de conceptos y teorías con el resto } \\
\text { de los miembros de la comunidad } \\
\text { Fomentar el establecimiento de mecanismos de distribución } \\
\text { de la información y el conocimiento generado entre los } \\
\text { miembros de la comunidad }\end{array}$ \\
\hline
\end{tabular}

Fuente: Elaboración propia. 
La primera competencia puede ser establecida como condición necesaria para el momento de la intersubjetividad, mientras que la segunda, esencialmente, nos conduciría a ese momento.

El momento del retorno sobre sí mismo involucraría las siguientes competencias y atributos.

\section{Cuadro 4}

Competencias y atributos referidos al momento del retorno sobre sí mismo

\begin{tabular}{|c|c|}
\hline Competencia & Atributos \\
\hline \multirow[t]{5}{*}{$\begin{array}{l}\text { Crear y mantener procesos de } \\
\text { autorregulación }\end{array}$} & $\begin{array}{l}\text { Aplicar el ciclo planificación, actuación y reflexión a } \\
\text { situaciones específicas de formación }\end{array}$ \\
\hline & $\begin{array}{l}\text { Realizar la planeación, el autoseguimiento y la gestión de } \\
\text { recursos para la consecución de sus propósitos }\end{array}$ \\
\hline & $\begin{array}{l}\text { Elaborar autoinformes sobre su propia experiencia auto- } \\
\text { rreguladora }\end{array}$ \\
\hline & $\begin{array}{l}\text { Utilizar estrategias de identificación y gestión del conoci- } \\
\text { miento adquirido }\end{array}$ \\
\hline & $\begin{array}{l}\text { Utilizar el seguimiento y la evaluación para obtener cono- } \\
\text { cimiento del proceso seguido }\end{array}$ \\
\hline \multirow{3}{*}{$\begin{array}{l}\text { Desarrollar un pensamiento } \\
\text { Creativo }\end{array}$} & Construir visiones alternativas de una situación dada \\
\hline & $\begin{array}{l}\text { Crear mapas perceptuales para la identificación de los } \\
\text { diferentes planos de una situación dada }\end{array}$ \\
\hline & $\begin{array}{l}\text { Solución de problemas de manera no convencional para } \\
\text { desarrollar diferentes vías de solución }\end{array}$ \\
\hline \multirow[t]{6}{*}{$\begin{array}{l}\text { Construir disertaciones con base } \\
\text { en una lógica argumentativa }\end{array}$} & $\begin{array}{l}\text { Conocer qué es un argumento y los diferentes tipos de } \\
\text { argumentos }\end{array}$ \\
\hline & $\begin{array}{l}\text { Crear modelos para la presentación de textos argumenta- } \\
\text { tivos }\end{array}$ \\
\hline & Definir tesis para la construcción de textos argumentativos \\
\hline & $\begin{array}{l}\text { Construir argumentos para sustentar la tesis de un trabajo } \\
\text { argumentativo }\end{array}$ \\
\hline & Buscar información para fundamentar los argumentos \\
\hline & $\begin{array}{l}\text { Construir una línea discursiva para la presentación verbal } \\
\text { de su tesis }\end{array}$ \\
\hline
\end{tabular}

Fuente: Elaboración propia.

Las dos primeras competencias se plantean como condiciones necesarias para este momento, mientras que la tercera permitiría cerrar el ciclo de formación a través de la reconstrucción. 
Algunas sugerencias bibliográficas. Favorecer el desarrollo de estas competencias no se puede lograr a través de una práctica docente tradicional, por lo que se hace necesario que los formadores de docentes realicen algunos cambios en sus procesos de enseñanza. En ese sentido se recomiendan los siguientes métodos, o metodologías, que creo permitirían establecer una fuerte correspondencia con las competencias ya definidas:

- Métodos para fomentar el pensamiento crítico (Espíndola 2000)

- La enseñanza estratégica (Díaz Barriga y Hernández 1998)

- Teoría del Diseño Educativo basado en el aprendizaje autorregulado (Corno y Randi 2000)

- La resolución de problemas en colaboración (Miller 2000)

- El aprendizaje cooperativo en el aula (Johnson, Johnson y Holubec 1999)

- Comunidades de aprendizaje en el aula: una reconceptualización de la práctica de la enseñanza (Bielaczyc y Collins 2000).

Estos métodos, o metodologías, necesariamente deben integrarse en una estrategia global que permita que la sinergia que se genere a partir de su interrelación coadyuve al logro de la formación.

\section{A MANERA DE CIERRE}

La formación profesional por competencias suele ser un campo que generalmente se visualiza como árido, por el abuso del discurso prescriptivo de carácter ateórico y pragmático, y caótico, por la multiplicidad de líneas discursivas y su falta de integración.

En este contexto discursivo es que se presenta el presente trabajo que intenta ofrecer, a partir de una conceptualización comprehensiva de la formación, una línea discursiva crítica que supere la visión pragmática e instrumental prevaleciente en el campo.

Para lograr ese objetivo se dividió este trabajo en tres apartados: en el primero se planteó la limitante central del enfoque de formación por competencias (su carácter pragmático, utilitarista e inmediatista), en el segundo apartado se construyó una conceptualización comprehensiva de la formación, de claro tinte hegeliano, y a partir de ella, en el tercer apartado se realizó una propuesta de competencias genéricas para la formación de docentes.

Por último, y aunque parezca innecesario, quiero enfatizar que la propuesta de competencias genéricas para la formación de docentes, que se realiza en este trabajo, solamente puede adquirir pleno sentido bajo una conceptualización comprehensiva de la formación.

\section{REFERENCIAS}

Abbagnano, N. y A. Visalberghi (1993). Historia de la Pedagogía. México: FCE.

Aguerrondo, I. y P. Pogré (2001). Las instituciones de formación docente como centros de innovación pedagógica. Buenos Aires, Argentina: Troquel.

Aimerd, P. (1987). El lenguaje del niño. México: FCE. 
Alanis Huerta, A. (2000). Formación de formadores. México: Trillas.

Barraza Macías, A. (2004). La identidad de las instituciones de educación superior en el horizonte de la postmodernidad, disponible en URL: www.ilustrados.com

Beltrán Ugarte, F. (2000). La experiencia de Conalep en la educación basada en normas de competencia". En Competencia laboral y educación basada en normas de competencia de Antonio Argüelles (comp.), México, Limusa: SEP, CNCCL y CONALEP.

Bielaczyc, C. y A. Collins. (2000). "Comunidades de aprendizaje en el aula: una reconceptualización de la práctica de enseñanza”. En Diseño de la instrucción. Teorías y modelos. Madrid, España, Santillana.

Casanova, F. (2003). Formación profesional y relaciones laborales. Montevideo, Uruguay: CINTERFOR.

Cejas, E. y J. Pérez. (2003). Un concepto muy controvertido: competencias laborales, disponible en URL: http://www.monografias.com

Cejas Yanes, E. (2004). La formación por competencias laborales: proyecto de diseño macrocurricular para la especialidad de farmacia industrial, disponible en URL: http://www.ilustrados. com/documentos/competencias.doc

Cejas Yanes, E. (2004). Un análisis de la definición de competencia laboral, disponible en URL: http://www.monografías.com

CIDEC. (2004). Competencias profesionales. Enfoques y modelos a debate. San Sebastián, España, Gobierno Vasco y Fondo Social Europeo.

Corno, L. y J. Randi. (2000). ¿Una teoría del diseño educativo basado en el aprendizaje autorregulado. En Diseño de la instrucción. Teorías y modelos. Madrid, España: Santillana.

Davini María, C. (1997). La formación docente en cuestión: política y pedagogía, Barcelona, España: Paidós.

Díaz Barriga Arceo, F. y G. Hernández Rojas (1998). Estrategias docentes para un aprendizaje significativo, México: McGraw-Hill.

Espíndola Castro, J. L. (2000). Reingeniería educativa. El pensamiento crítico: cómo fomentarlo en los alumnos. México: Pax.

Ferry, G. (1990). El trayecto de la formación. Los enseñantes entre la teoría y la práctica, México, Paidós.

Ferry G. (1997). Pedagogía de la formación. Buenos Aires, Argentina: Ediciones Novedades Educativas y Universidad de Buenos Aires.

Fletcher, S. (2000). Diseño de capacitación basada en competencias laborales. México: Panorama.

Forgas, J. (2003). Diseño curricular por competencias: Una alternativa para la formación de un técnico competente. Ciudad de La Habana, Cuba: Curso 14 Pedagogía.

Gadamer Hans, G. (1996). Verdad y Método, Tomo I, Fundamentos de una Hermenéutica Filosófica. Salamanca, España: Sígueme.

García Jesús, M., S. Rubio Valdehita y J. Lillo Jover. (2003). Qué es la psicología del trabajo, Madrid, España: Biblioteca Nueva.

Gilbert, R. (1977). Las ideas actuales en pedagogía. México: Grijalbo.

Ginzo, A. (1998). Hegel y el problema de la educación. En Escritos Pedagógicos de G. W. F. Hegel. México: FCE:

Gonczi, A. y J. Athanasou. (2000). Instrumentación de la educación basada en competencias. Perspectivas de la teoría y la práctica en Australia. En Competencia laboral y educación basada en normas de competencia de Antonio Argüelles (comp.). México: Limusa, SEP, CNCCL y CONALEP.

Guillén Gestoso, C. y R. Guil Bozal. (1999). Psicología del trabajo para relaciones laborales. Madrid, España: McGraw-Hill.

Habermas, J. (1993). Teoría de la acción comunicativa: complementos y estudios previos. México: REI. 
Habermas, J. (2001). Teoría de la acción comunicativa, I. Madrid, España: Teurus.

Hanson, M. (2000). Lecciones y experiencias del desarrollo de la educación y la capacitación basadas en competencias en el Reino Unido. En Competencia laboral y educación basada en normas de competencia de Antonio Argüelles (comp.). México: Limusa, SEP, CNCCL y CONALEP.

Honoré, B. (1980). Para una teoría de la formación. Madrid, España: Narcea.

Huerta Amezola, J., I. S. Pérez García y A. R. Castellanos Castellanos. (2000). Desarrollo curricular por competencias profesionales integradas. Revista Educar 13.

Imbernón, F. (1997). La formación del profesorado. Barcelona, España: Paidós.

INSAFORP. (2001). Fundamentos de la formación profesional por competencias, Salvador, El Salvador: Autor.

Johnson David, W., T. Johnson Roger y J. Holubec Edythe. (1999). El aprendizaje cooperativo en el aula. Buenos Aires, Argentina: Paidós.

Kobinger, N. (2000). El sistema de formación profesional y técnica por competencias desarrollado en Quebec. En Competencia laboral y educación basada en normas de competencia de Antonio Argüelles (comp.). México: Limusa, SEP, CNCCL y CONALEP.

Leontiev, A. N. (1995). Artículo de introducción sobre la labor creadora de L. S. Vygotski. En Obras escogidas, tomo I de Liev Semiónovich Vygotski. Madrid, España: Visor.

Malpica Jiménez, M. C. (2000). El punto de vista pedagógico. En Competencia laboral y educación basada en normas de competencia de Antonio Argüelles (comp.). México: Limusa, SEP, CNCCL y CONALEP.

Miller Nelson, L. (2000). La resolución de problemas en colaboración. En Diseño de la instrucción. Teorías y modelos. Madrid, España: Santillana.

Moreno Palomares, A. (2005). El Concepto de formación. Disponible en http://www.paneldecomunicacion.net/aurelio/articulos/concepto.PDF

Morfin, A. (2000). La nueva modalidad educativa: Educación Basada en Normas de Competencia. En Competencia laboral y educación basada en normas de competencia de Antonio Argüelles (comp.). México: Limusa, SEP, CNCCL y CONALEP.

Ruiz Iglesias, M. (2005). La formación de competencias en educación: una mirada detenida en las competencias de la profesión docente. Durango, Documento de trabajo en el Doctorado en Ciencias de la Educación del Instituto Universitario Anglo Español.

Saluja, S. (2000). La capacitación basada en competencias en el Reino Unido. En Competencia laboral y educación basada en normas de competencia de Antonio Argüelles (comp.), México: Limusa, SEP, CNCCL y CONALEP.

Schraml Walter, J. (1970). Initiation a la pedagogie psychanalytique. Salvador: Casterman.

Souto, M. (1999). Grupos de formación. En Grupos y dispositivos de formación, de Marta Souto, Jean Marie Barbie, Mabel Cattaneo, Mirta Coronel, Laura Gaidulewics, Nora E. Goggi y Diana Mazza. Buenos Aires, Argentina: Ediciones Novedades Educativas/Universidad de Buenos Aires.

Spencer, Ll. y A. Krauze. (2002). Hegel para principiantes. Buenos Aires, Argentina: Era Naciente.

Tejada, Fernández, J. (2005). El trabajo por competencias en el prácticum: cómo organizarlo y cómo evaluarlo. En Revista Electrónica de Investigación Educativa, Vol. $7 \mathrm{~N}^{\circ} 2$.

Yurén Camarena, M. T. (1993). Sujeto, eticidad y educación en la obra de Hegel. México: Universidad Pedagógica Nacional.

Yurén, Camarena M. T. (2000). Formación y puesta a distancia. México: Paidós Educador. 